\title{
A TASK-DRIVEN DISASTER DATA LINK APPROACH
}

\author{
L.Y. Qiu ${ }^{a}$, Q. Zhu ${ }^{\text {b, c }}$, J.Y. Gu ${ }^{\text {a }}$ Z.Q. Du ${ }^{\text {a, d, } *}$ \\ ${ }^{a}$ State Key Laboratory of Information Engineering in Surveying, Mapping and Remote Sensing, Wuhan University, 430079 Wuhan, \\ China-qiu_linyao@163.com, duzhiqiang@whu.edu.cn, gujieye@126.com \\ ${ }^{\mathrm{b}}$ State-province Joint Engineering Laboratory of Spatial Information Technology for High-Speed Railway Safety, Southwest \\ Jiaotong University, 610000, Chengdu, China-zhuq66@263.net \\ ${ }^{\mathrm{c}}$ Faculty of Geosciences and Environmental Engineering, Southwest Jiaotong University, 610000, Chengdu, China - \\ zhuq66@263.net \\ ${ }^{\mathrm{d}}$ Collaborative Innovation Center of Geospatial Technology, 430079 Wuhan, China -duzhiqiang@whu.edu.cn
}

KEY WORDS: Emergency Task, Ontology, Disaster Data Management, Semantic Mapping, Spatial-temporal Correlation, Data Link

\begin{abstract}
:
With the rapid development of sensor networks and Earth observation technology, a large quantity of disaster-related data is available, such as remotely sensed data, historic data, cases data, simulation data, disaster products and so on. However, the efficiency of current data management and service systems has become increasingly serious due to the task variety and heterogeneous data. For emergency task-oriented applications, data searching mainly relies on artificial experience based on simple metadata index, whose high time-consuming and low accuracy cannot satisfy the requirements of disaster products on velocity and veracity. In this paper, a task-oriented linking method is proposed for efficient disaster data management and intelligent service, with the objectives of 1) putting forward ontologies of disaster task and data to unify the different semantics of multi-source information, 2) identifying the semantic mapping from emergency tasks to multiple sources on the basis of uniform description in 1), 3) linking task-related data automatically and calculating the degree of correlation between each data and a target task. The method breaks through traditional static management of disaster data and establishes a base for intelligent retrieval and active push of disaster information. The case study presented in this paper illustrates the use of the method with a flood emergency relief task.
\end{abstract}

\section{INTRODUCTION}

The number of incidents and magnitude of natural disasters worldwide have increased significantly due to climate changes in recent years (Ding et al., 2014; Iwata et al., 2014; Neumayer et al., 2014). A number of natural disasters (e.g., South Asia Tsunami, China Earthquake, Haiti Earthquake and Tohoku Earthquake) stroke across the globe, killing hundreds and causing billions of dollars in property and infrastructure damage (Grolinger et al., 2013).

Facing the urgent need for disaster mitigation, understanding how to enhance the capacity of effective monitoring, early warning and emergency response have become major challenges all around the world. On one hand, the amount of information and types of data related to disaster has increased greatly. Disaster data, including remote sensing images, history data, previous incidents records, simulation data basic geographic data and disaster assessment products possess velocity, variety and veracity features converted from singleness and small amount. They put forward a higher requirement for integration, processing and analysis (Grolinger et al., 2013). On the other hand, government agencies in different levels and individual organizations master various data resources and take different disaster relief functions. In order to achieve good cooperation and collaboration in disaster management, the most effective data should be sent promptly to the most needed actors (Borkulo et al., 2006). In recent years, various types of sensors widely deployed in disaster monitoring network make it possible to continuously access disaster big data with high spatial-temporal resolution and increasingly rich attribute information, which provides important support for enhancing capabilities of disaster emergency response. However, fast and easy acquisition and generation of heterogeneous data has exceeded existing ability of data management. The main reasons are as follows: 1) most existing disaster management systems operate in a typical passive data-centric mode (Ding et al., 2014). The functions and purpose of disaster information service are typically singular and direct, which could rapidly satisfy specific user needs, but will not fit the needs of the actual disaster management tasks of other user communities. The functions also do not generate products with high accuracy and veracity when the needed data source is limited or not accessible. 2) Current efforts to integrate geographic information data have been restricted to keyword-basedmatching Spatial Information Infrastructure (SII) (Li et al., 2007). SII supports the discovery and retrieval of distributed geospatial data sources and geographic information services by providing catalogue services and syntactic interoperability standards (Lutz, 2007), but spatial-temporal characteristics of data (e.g., the spatial distribution of the cloud in a multispectral remote sensing image) are hidden inside the data file. Moreover, lack of a semantic association among multi-source heterogeneous data brings a passive result that the knowledge and discipline of the disaster are hardly found automatically. 3) Recent disaster data retrieval mainly relies on querying with keywords of metadata passively. The artificial experience plays an important role in finding available data because there is short

\footnotetext{
* Corresponding author: DU Zhiqiang. E-mail: duzhiqian@whu.edu.cn.
} 
of mechanism of automatically discovering related data and disaster knowledge for computer reasoning (Fan and Zlatanova, 2011). In practice, trivial and time-consuming operations to integrate various resources have cost most the manual resources rather than improving the decision-making (Laniak et al., 2013). This is why most existing disaster management systems have been of limited use (Leskens et al., 2014) and resources cannot be fully utilized (Demir and Krajewski, 2013; Zhishan et al., 2012). This paper proposes a task-oriented disaster information link method, in which disaster emergency tasks are regarded as a key semantic factor to restrain, associate and aggregate spatial-temporal data.

Here, we discuss the challenge of managing disaster data to support various task processing in emergency response contexts. The paper is organized as follows: "Related Work" section presents related work on applying semantic-related technology and ontology for spatial data and emergency response. The section titled "Task and data ontologies for disaster management" firstly analyzes types and features of emergency tasks in disaster management and puts forward an ontology model describing them. Then it describes the semantic features of disaster data in regards to attribute, space-time and statistics. "Semantic mapping of task and data" discusses a map between characteristics of emergency tasks and disaster data in scale, attribute and spatial-temporal level and proposes task-oriented multi-dimensional data characteristics to analyse task preference to different data sets. The "Implementation" section introduces a case study illustrating how to aggregate data in a multilevel way to find the right data for a specific emergency task in storm-flood disaster chain. Finally, we conclude the article in "Conclusions and future work" section.

\section{RELATED WORK}

\subsection{Related work on the semantic technology in disaster data management}

As mentioned earlier, the existing disaster-related data is extremely heterogeneous and different vocabulary could be used in different sources. The reason why semantic-related technology is employed is that they can be used to identify and associate semantically-corresponding concepts with disasterrelated information so heterogeneous data can be integrated and ingested (Hristidis et al., 2010). Many previous studies have discussed the importance of semantic-related technology for solving problems in geographical information systems (Cohn, 1997; Guarino, 1998). Cohn (1997) proposed that the humancomputer interaction in GIS should be more concise and accurate than it is currently. Currently, aiming at resolving semantic diversity generating adverse effect on data management and achieving semantic interoperation among heterogeneous data, spatial semantic description has been used in disaster data management (Fan and Zlatanova, 2011; Li et al., 2007; Zhu et al., 2009; Schulz et al., 2012; Silva et al., 2013). Schulz (2012) and Silva (2013) established description of data by Linked Open Data (LOD). Based on semantic web knowledge, they adopted RDF (Resource Description Framework) to define standard and exchangeable data format for semantic annotation of disaster knowledge. LOD is considered an effective tool that could convert data relations to information computers could process, promoting automatic finding and reasoning of disaster knowledge (Foster and Grossman, 2003; Lausch et al., 2014). Michalowski (2004) also applied the Semantic Web technology to develop a Semantic Web-enabled management system. Such a system allows efficiently querying distributed information and effectively converting legacy data into more semantic representations (Michalowski et al., 2004). Zhu (2009) analysed challenges of intricate semantics in remote sensing information systems and proposed a hierarchical semantic restrain model as a uniform semantics description model. The connection between user semantics, data and processing services is established as basics of semantic reasoning in discovery, selection and composition of data and service.

\subsection{Related work on ontology in disaster data management}

Compared with the semantic methods mentioned, ontology has stronger semantic integrity and supports uniform description from data definition to operation. This is useful for automatic finding and mining of data (Babitski et al., 2009; Klien et al., 2006). Guarino (1998) analyzed the importance of the ontology concept in GIS system. However, he only proposed a possible ontology structure without attempting to implement it. Some researches provided conceptual structures of ontology in disaster management (Chatterjee and Matsuno, 2005; Li et al., 2009; Xu et al., 2009). Chatterjee and Matsuno (2005) discussed the necessity of using the ontology to solve the linguistic differences. Li (2009) proposed an ontology-based architecture for geo-objects in disaster systems. Xu (2009) also suggested building an ontology-based emergency response plan. Some researchers have studied specific ontology methods for semantic description (Huang and Yan, 2013; Wang et al., 2007; Yang et al., 2013). Huang (2013) proposed disaster domain ontology including hazard-affected body, disaster-inducing factors, inducing environment, disaster events and built connections among them by ontology. The model was experienced in disaster processing estimation and prediction. Babitski (2009) defined ontology of disaster damage, resource and the relations between them, so that available data resource could be quickly found while facing a certain assessment task. Wang (2007) put forward a spatial geographic ontology by analysing objects, relation and data in space. Such a description effectively presents hierarchical structure and semantic relation of spatial information. Yang (2013) developed a kind of task ontology, dividing task process from aspects of function, organization, spatial-temporal scale and complexity of calculation.

Although current semantic methods resolve problems of integration in disaster data management, most of them manage limited types of data and the semantic restraints or correlation on heterogeneous data are simple. Thus, a mature ontologybased data correlation method is required so that can both integrate heterogeneous data from different sensors and support automatic querying and reasoning functions.

\section{TASK AND DATA ONTOLOGIES FOR DISASTER MANAGEMENT}

\subsection{A task ontology for emergency workflow}

The need for up-to-date geospatial data in emergency situations is now widely recognized. Emergency responders may not be familiar with data standards or the appropriateness of certain data sets for a particular task. However, due to the critical nature of emergency response, responders rarely have time to sift through extensive query results and will not re-think what data sources and specific data characteristics are needed each time they face a task. Thus, it is worthwhile to formally delineate tasks and their relationships to types of data sources (Wiegand and García, 2007). 


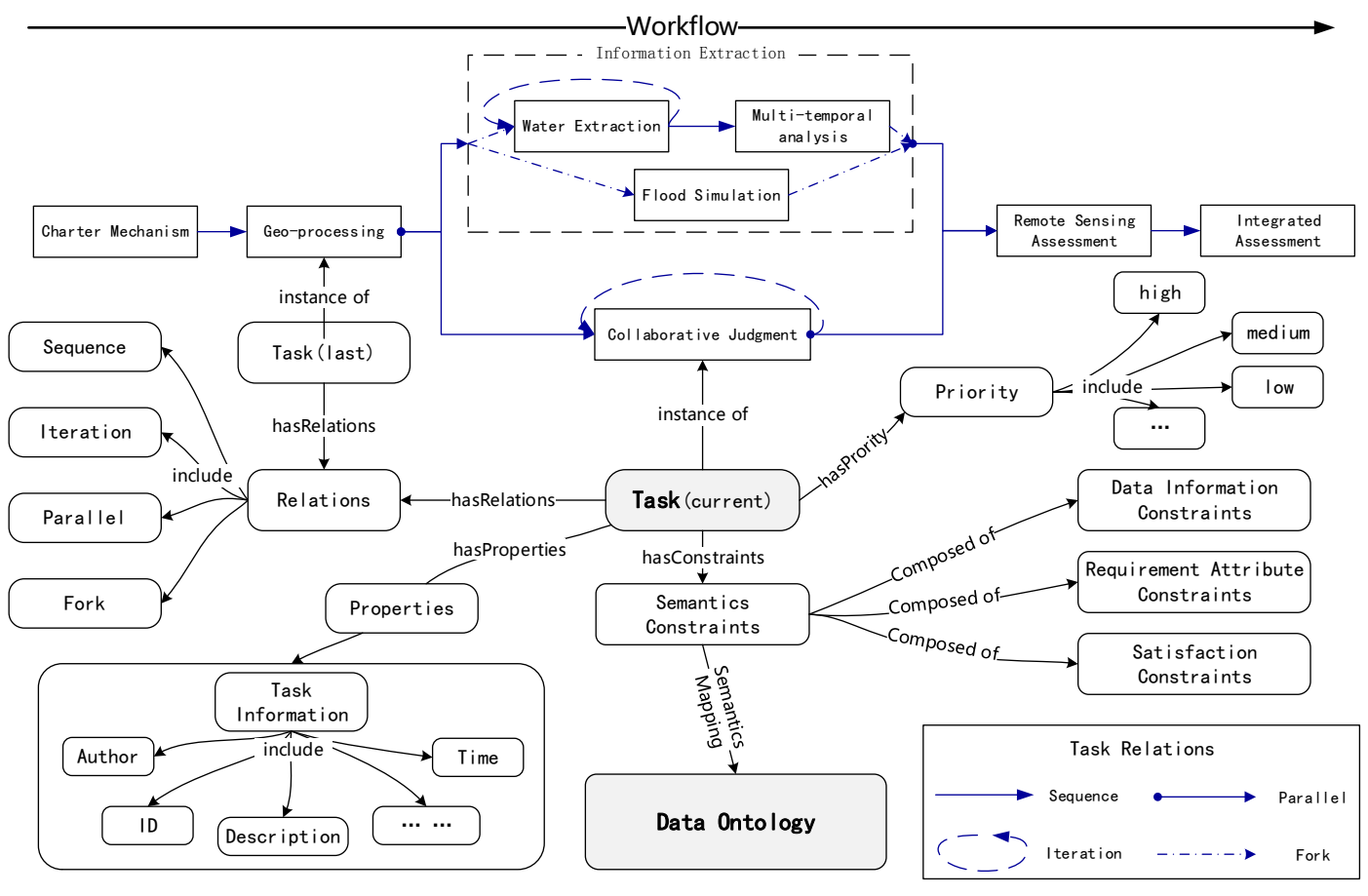

Figure 1. Structure of a task ontology in an emergency response workflow

Ontology is a description, in a formal, machine-readable format, that expresses concepts including the types of entities, attributes, relationships, and values found in a domain. Ontology can represent the semantics of emergency tasks and disaster data, thus helping create connections between them for emergency response processes. Therefore it contributes to the knowledge of workflow processing and task demand. In this paper, the task ontology in supporting emergency response is abstracted as a quintuple:

$$
O_{T}=<C, R, S, P, I>
$$

$C$ represents task basic information based on functional and non-functional properties; $R$ represents the relationships between a task entity and others in an emergency workflow; $S$ represents the semantic restraints of a task demand. $P$ represents the priority of task execution and data retrieval in the emergency workflow; I represents the instances of tasks.

Taking a flood response workflow of the National Disaster Reduction Center in China (NDRCC) as an example, Figure 1 shows the ontological entities and relations of disaster tasks. A set of connections composes a directed graph that specifies how the task works. The whole emergency workflow contains a group of tasks such as charter mechanism based acquisition of satellite imageries, geo-processing of data, information extraction, collaborative judgment, remote sensing assessment and integrated assessment. Each task is an instance of an ontology entity. The relations including sequence, parallel, interaction and fork rules the logic order of task execution process. As the process is developing, the priority of a task is changing dynamically in real time, which further influences the queue of data retrieval and preparation. For instance, when the task of collaborative judgment following geo-processing proceeds, its execution priority is higher than its successors (such as remote sensing assessment) but the same as information extraction because they are parallel. The semantic restraints describing the feature of task demand are composed by three parts: data information, requirement attributes and satisfaction. Data information confines the basic feature of input data including resolution, timeliness, types of sensors and spatial system. Requirement attributes describe preference and selection rules to data in the background of a certain disaster. Satisfaction represents the quality of task output influenced by data quality, environmental factors, response speed and overlap extent of data with the target area. The task ontology representing the function, attributes, process and need of tasks is a precondition to link task and data. It is presented as a RDF/OWL (Web Ontology Language) file (http://www.semanticweb.org/dell/ontologyies/tasks/task.owl). Some snippets of the file are listed in Table 1. Hereafter, ontologies are presented in protégé for clarity.

Table 1. Snippets of the task ontology file in Turtle

<!--http://www.semanticweb.org/dell/ontologies/task\#Atomic--> <owl:Class rdf:about="\&task;Atomic">

$<$ rdfs:subClassOf rdf:resource="\&task;TaskType"/>

$</$ owl:Class $>$

$<$ !-- http://www.semanticweb.org/dell/ontologies/task\#AtomicTask $->$

<owl:Class rdf:about="\&task;AtomicTask">

$<$ rdfs:subClassOf rdf:resource="\&task;Task"/>

$</$ owl:Class $>$

$<!-$

http://www.semanticweb.org/dell/ontologies/task\#CompositTask -$>$

<owl:Class rdf:about="\&task;CompositTask">

$<$ rdfs:subClassOf rdf:resource="\&task;Task"/>

$</$ owl:Class $>$

$<!--$ http://www.semanticweb.org/dell/ontologies/task\#Composite -

$\rightarrow$

<owl:Class rdf:about="\&task;Composite">

$<$ rdfs:subClassOf rdf:resource="\&task;TaskType"/>

$</$ owl:Class $>$ 


\subsection{An ontology of disaster data}

A success of disaster data management could be described as "getting the right resources to the right place at the right time; to provide the right information to the right people to make the right decisions at the right level at the right time $(\mathrm{Xu}$ and Zlatanova, 2007)." However, semantic heterogeneity of the spatial data remains one of the biggest challenges in disaster data management. Especially, as acquisition of multi-source data including remote sensing images, history data, case data, simulation data, basic geographic data and disaster assessment product has become increasingly easy and fast, metadata catalogs based data management can neither unify heterogeneous semantics nor explicitly represent correlation of various data. So data ontology is designed to solve the problem through the integration of disaster data and a triple is constructed for its description:

$$
O_{D}=<T, F, I>
$$

$T$ represents the type classification of disaster data by defining a two-tuples composed of category and format. Category describes the conceptual classification, like observed data and history data, while format denotes specific file pattern, such as geotiff, img and shpfile (as shown in Figure 2). $F$ represents the apparent and potential features of data from three aspects: attribute, space-time and statistics. Attribute contains inherent nature of data, which is obtained from data itself including spatial and temporal resolution, spatial reference and spectrums. Space-time describes spatial-temporal information including velocity of data acquisition and scope of the area covered by data. Such information is commonly obtained from record or calculation. Statistics show the rules and knowledge about data usage, such as the operating frequency of the data while facing a specific task. Then $I$ represents the instances of data.

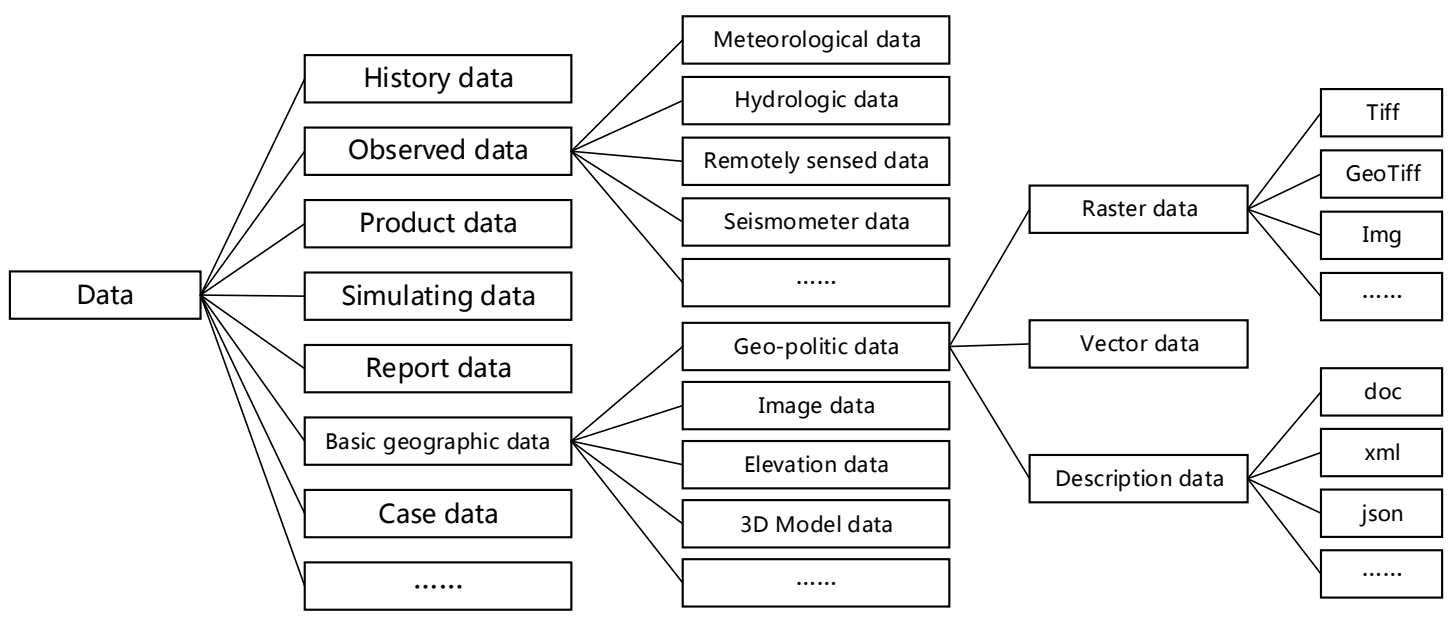

Figure 2. Disaster data classification

The relations of different data instances can be described in two aspects. For the data with disparate types, the correlation of them is described by statistics features in a common application environment, like the co-occurrence of heterogeneous data adopted in similar history cases. For data of the same type, the correlation is built by calculating the similarity of spatial and temporal features. The similarity is calculated by the following formula:

$$
\operatorname{Sim}_{\text {case }(i, j)}=w_{t} \times \alpha^{\left|D_{j}-D_{i}\right|}+w_{s} \times \beta^{-\ln \frac{\left.\operatorname{Min}_{\text {(Area }}, \text { Area }_{j}\right)}{\operatorname{Max}\left(\text { Area }_{i}, \text { Area }_{j}\right)}}
$$

$w_{t}$ is the weight of temporal similarity while $w_{s}$ is the weight of spatial similarity. The sum of $w_{t}$ and $w_{s}$ equals 1 , but their specific values rely on the task need. For example, $w_{t}$ in temporal series analysis is higher than that in other tasks. $\alpha$ and $\beta$ are two decay factors ranging from 0 to $1 .\left|D_{j}-D_{i}\right|$ represents the absolute interval value of two dates. Min and Max respectively means the overlap area of two sets of data and union area of minimum bounding box containing them. When the calculation is close to or equals 1 , the degree of connection between two data sets are strong while if the value approximates 0 , they have a weak connection. So the data ontology not only unifies the semantic description of heterogeneous data, but also offers the correlation method to automatically find other related data resources in the searching process.

\section{SEMANTIC MAPPING OF TASK AND DATA}

Due to a lack of semantic association between tasks and data in traditional disaster data management, the determination of which data source is the most appropriate for a specific task, as implicit knowledge, could not be commonly applied. However, a unified description containing task and data is complex and unnecessary because they belong to different domains and their own respective composition. Thus expressions with common semantic terms could neither highlight each feature characteristic nor help the system increase automatic understanding and analysis to disaster knowledge. Clearly, one should be able to connect tasks with data. Based on the ontologies, in order to build connections between task and data and further, convert task needs to specific data query filters, a mapping from semantics constraints of task ontology to that of data ontology is designed, which is expressed as:

$$
O_{T}(S) \rightarrow O_{D}(F)
$$

As shown in Figure 3, the mapping relation contains almost all the task needs and features of disaster data, and there are several mapping types, including one-to-one, one-to-many and many-to-many between them. A further classification 
including attribute, space-time and scale is built on the base of mapping relations. The attribute level describes some indicators showing which data set is more suitable for a specific task by analysing the statistics of data usage in similar historical cases. For instance, the high co-occurrence and adopting frequency represents the importance of a data source to a task. So mapping relations in the attribute level could describe what kind of data source is the most suitable to current emergency process and help the system analyse the feature for automatic retrieval. The spatial-temporal level contains the direct correlation of tasks and data like task requirement on coordinate system, spatial reference and coverage area of data, which could filter the inappropriate data source and choose the potential source when the attribute-based retrieval does not find default suitable targets. The scale offers some flexible relevance factors like resolution of data. Such factors support analysing the correlation of task and potential data sets by calculating the degree of satisfaction and finally, a list of data based on quantitative estimation of correlation could be provided for task operators.

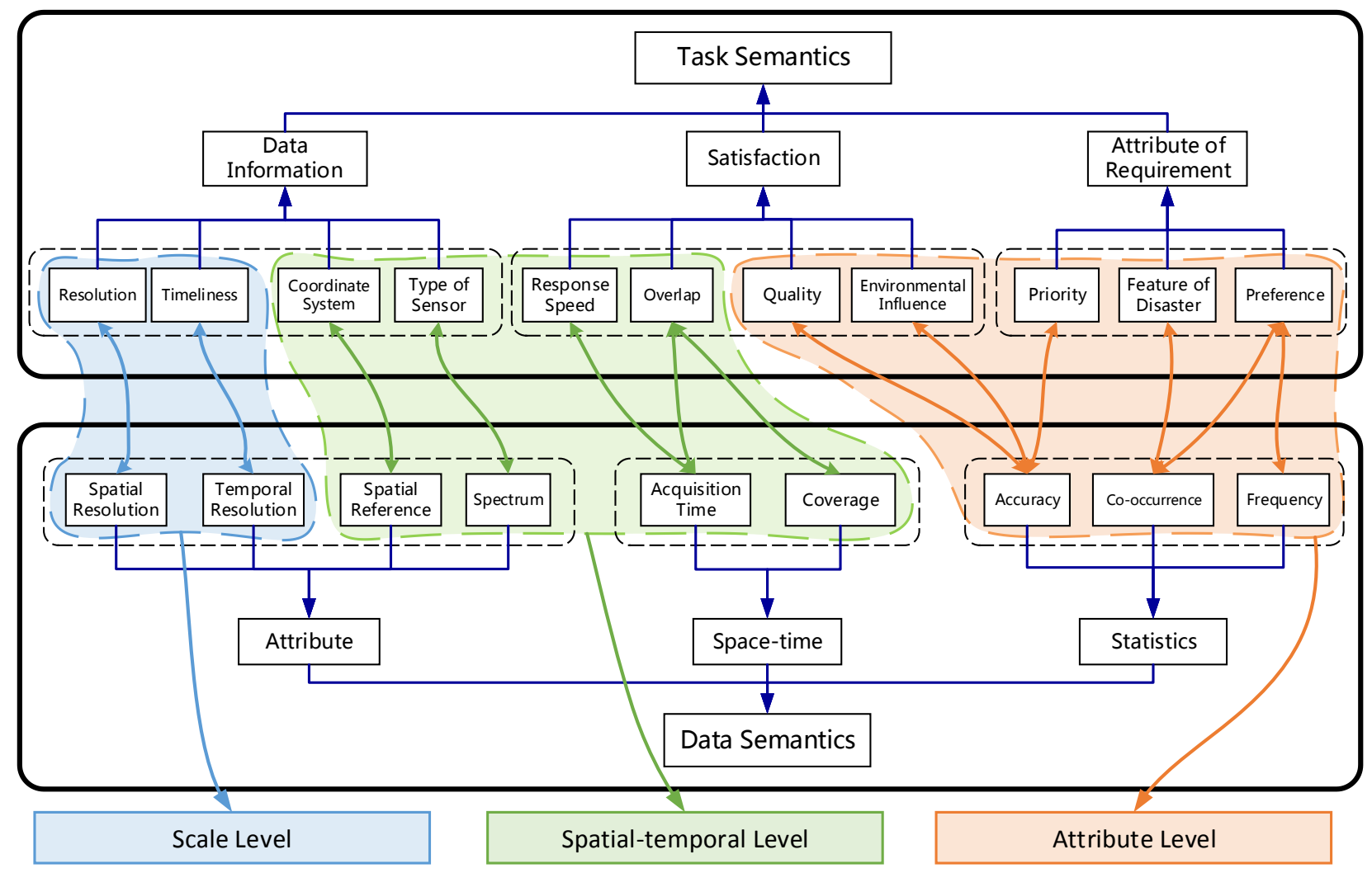

Figure 3. Semantic mapping between task and data

So far, the task-oriented disaster data link method proposed in this paper contains three parts. Firstly, the relation of tasks mentioned in section 3 builds the correlation of different tasks in a workflow, so the system could query and compose a workflow with needed tasks by itself. This sets a goal and order for automatic data preparation. Secondly, the similarity of data with the same category is calculated in spatial and temporal aspects. So while a data set well satisfying the running task is found, continuously some other related data could be searched by the system actively, accompanied with a quantitative analysis on relevance between potentially useful data and the target. Such operation helps users find more suitable resources for a specific task in advance. Thirdly, a classified mapping from task semantics to data semantics is set up for connecting emergency task and disaster data. Then the mapping drives a multi-level-based searching and filtering process to actively offer users the most suitable data satisfying target task quickly and precisely.

\section{IMPLEMENTATION}

A prototype system for building custom task flow and searching suitable data is developed and integrated in a SOA (ServiceOriented Architecture) based business-operation monitoring and management platform deployed in NDRCC, which is used for monitoring and managing disaster mitigation in whole emergency response period. Once users establish a new disaster task (as shown in Figure 4 (a)), by comparing factors like type, level and location of disaster with historical cases, the prototype system could set up a series of executable workflow and support man-machine manner (drag, drop and compose) to modify the process chain (as shown in Figure 4 (b)). We used the real-time emergency response task of the flood in Fushun, China in 2013 as an example. There are more than 40 typical flood events from the year of 2000 stored in the history database of NDRCC. Therefore, through selecting atomic tasks frequently used in similar history tasks, the system first composes a new workflow. Users could change it on the interface shown if necessary. Then the priority of each task is distributed relying on its location in the process chain. After that, data preparation including 
retrieval and selection process starts according to the priority rank.

Firstly, the system loads and parses RDF file of current task ontology using Jena and a list of related data type is created. Taking integrated assessment process for instance, the type list of its needed data includes raster (post-disaster urban image of Fushun, flood figure), vector (administrative map, flooding extraction figure, distribution diagram of damaged infrastructures and houses) and text (yearbook of population statistics and economy statistics in Fushun, reported data from disaster area). Then system starts to traverse the list to find the most suitable data for each type. Secondly, according to the correlation of task and data in attribute level, the data with the most frequency usage in history case will be searched. For example, the post-disaster raster image is used as a background

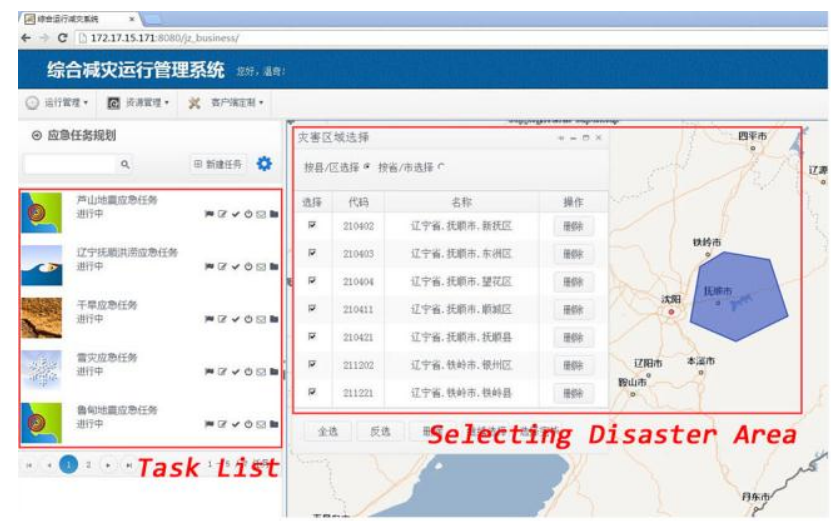

(a) Setting up a disaster task to show information of disaster area as rich as possible, the images of ZY-3 satellite was often chosen in this application, then the information will be obtain from RDF file directly. But if the ZY-3 images could not find it in the database, a further analysis to find potential right data proceeds. The system parses factors in spatial-temporal level to build a query condition, then images that can not satisfy Fushun flood in spatial reference, area and other conditions will be filtered and an available image set will be selected. Further, using formula (3), the system calculates the similarity of integrated assessment and each images in the data set in space, time and resolution and ranks them according to the correlation degree. Finally, a series of images labeled with relevance to the task will be arranged in data selection interface (shown as Figure 4 (c)) so users could choose the most suitable data for the assessment.

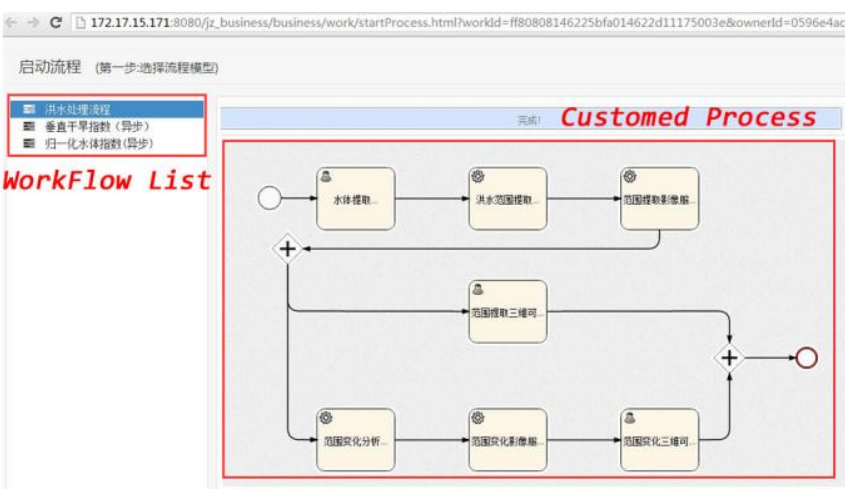

(b) Selecting workflow

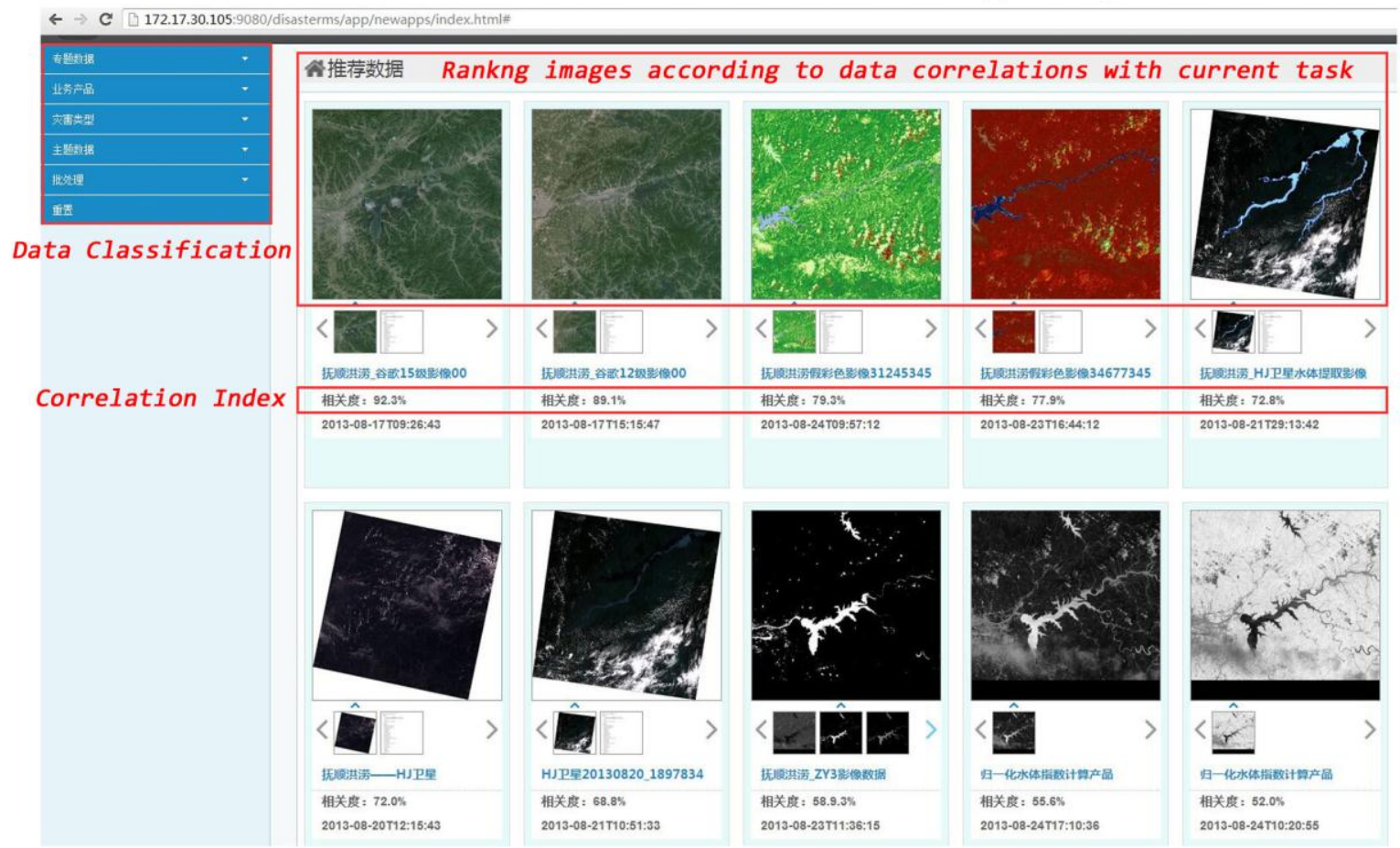

(c) Recommended data sets

Figure 4. The graphic interface of the prototype system

\section{CONCLUSION AND FUTURE WORK}

Compared to existing disaster data managing methods, there are several advantages to creating a task-oriented information link method using ontologies. Currently, searching for geospatial data can be overwhelming when one does know exactly which keywords to use. It can also be time-consuming to sift through undesirable results due to either poor keyword selection or bounding coordinate discrepancies within metadata. The method described here offers an innovative correlation method 
and lowers the complexity of man-machine interaction to find data.

In this study, ontologies for tasks and data sources are created independently and semantic mapping is set up between their features. The effort to create such a knowledge base is worthwhile because the independent ontologies and their association support semantic-related operations on spatial data, and helping users extract task-related information accurately. Then the analysis process of data searching is elaborated through introducing an emergency task scenario. The presented work is at an early stage and further research will focus on studying refining the statistical factors to take full advantage of historical cases and offering formulas to quantify the similarity between statistical factors and tasks.

\section{ACKNOWLEDGEMENTS}

This work was supported by the National Natural Science Foundation of China (Nos. 41171311, 41471320, 41471332), the National High Resolution Earth Observation System (the Civil Part) Technology Projects of China, and National High Technology Research and Development Program of China (2013AA122301).

\section{REFERENCES}

Chatterjee, R. and Matsuno, F., 2005. Robot description ontology and disaster scene description ontology: analysis of necessity and scope in rescue infrastructure context. Advanced Robotics, 19(8): 839-859.

Cohn, A.G., 1997. Qualitative spatial representation and reasoning techniques, KI-97: Advances in Artificial Intelligence. Springer, Berlin Heidelberg, pp. 1-30.

Demir, I. and Krajewski, W.F., 2013. Towards an integrated Flood Information System: Centralized data access, analysis, and visualization. Environmental Modelling \& Software, 50: $77-84$

Ding, Y. et al., 2014. An integrated geospatial information service system for disaster management in China. International Journal of Digital Earth(ahead-of-print), pp. 1-28.

Fan, Z. and Zlatanova, S., 2011. Exploring ontologies for semantic interoperability of data in emergency response. Applied Geomatics, 3(2): 109-122.

Foster, I. and Grossman, R.L., 2003. Data integration in a bandwidth-rich world. Communications of the ACM, 46(11): 50-57.

Babitski, G., Probst, F., Hoffmann, J. and Oberle, D., 2009. Ontology Design for Information Integration in Disaster Management. GI Jahrestagung, 154: 3120-3134.

Grolinger K, Capretz M, Mezghani E, et al. Knowledge as a service framework for disaster data management. Enabling Technologies: Infrastructure for Collaborative Enterprises (WETICE), 2013 IEEE 22nd International Workshop on. IEEE, 2013: 313-318.

Guarino, N., 1998. Formal ontology in information systems: Proceedings of the first international conference (FOIS'98), 46. IOS press, Trento, Italy.
Hristidis, V., Chen, S., Li, T., Luis, S. and Deng, Y., 2010. Survey of data management and analysis in disaster situations. Journal of Systems and Software, 83(10): 1701-1714.

Huang, F. and Yan, L., 2013. Reasoning of ontology model for typhoon disasters domain based on Jena. Journal of Computer Applications, 3(33): 771-775, 779.

Iwata, K., Ito, Y. and Managi, S., 2014. Public and private mitigation for natural disasters in Japan. International journal of disaster risk reduction, $7: 39-50$.

Klien, E., Lutz, M. and Kuhn, W., 2006. Ontology-based discovery of geographic information services-An application in disaster management. Computers, Environment and Urban Systems, 30(1): 102-123.

Laniak, G.F. et al., 2013. Integrated environmental modeling: A vision and roadmap for the future. Environmental Modelling \& Software, 39: 3-23.

Lausch, A., Schmidt, A. and Tischendorf, L., 2015. Data mining and linked open data - New perspectives for data analysis in environmental research. Ecological Modelling, 295: 5-17.

Leskens, J.G., Brugnach, M., Hoekstra, A.Y. and Schuurmans, W., 2014. Why are decisions in flood disaster management so poorly supported by information from flood models? Environmental Modelling \& Software, 53: 53-61.

Li, J., Zlatanova, S. and Fabbri, A.G., 2007. Geomatics solutions for disaster management. Berlin, Heidelberg, New York, Springer.

Li, B., Liu, J., Shi, L. and Wang, Z., 2009. A method of constructing geo-object ontology in disaster system for prevention and decrease, International Symposium on Spatial Analysis, Spatial-Temporal Data Modeling, and Data Mining. International Society for Optics and Photonics. 74923I-74923I.

Lutz, M., 2007. Ontology-based descriptions for semantic discovery and composition of geoprocessing services. Geoinformatica, 11(1): 1-36.

Michalowski, M. et al., 2004. Retrieving and semantically integrating heterogeneous data from the web. Intelligent Systems, IEEE, 19(3): 72-79.

Neumayer, E., Plümper, T. and Barthel, F., 2014. The political economy of natural disaster damage. Global Environmental Change, 24: 8-19.

Schulz, A., Döweling, S. and Probst, F., 2012. Integrating Process Modeling and Linked Open Data to Improve Decision Making in Disaster Management. Guest Editors, pp. 16.

Silva, T., Wuwongse, V. and Sharma, H.N., 2013. Disaster mitigation and preparedness using linked open data. Journal of Ambient Intelligence and Humanized Computing, 4(5): 591602.

Borkulo, V.E., Barboza, V.S., Dilo, A., Zlatanova, S. and Scholten, H., 2006. Services for emergency response systems in the Netherlands, Proceedings of the Second Symposium on Gi4DM, Goa, India, pp. 6.

Wang, Y., Gong, J. and Dai, J., 2007. Spatial Data Semantic Query Based on Ontology. Journal of Geomatics, 2(32): 32-34. 
Wiegand, N. and García, C., 2007. A Task - Based Ontology Approach to Automate Geospatial Data Retrieval. Transactions in GIS, 11(3): 355 - 376.

Xu, R., Dai, X., Yang, F. and Lin, P., 2009. Research on the construction method of emergency plan ontology based-on owl, The 2009 International Symposium on Web Information Systems and Applications, Nanchang, China, pp. 019-023.

$\mathrm{Xu}, \mathrm{W}$. and Zlatanova, S., 2007. Ontologies for disaster management response. Geomatics Solutions for Disaster Management. Springer, Berlin Heidelberg, pp. 185-200.

Yang, H., Lv, G. and Sheng, Y., 2013. Distributed Collaborative Geographic Modeling Task Decomposition Method Based on HTN Planning. Acta Geodaetica et Catrtographica Sinica, 42(3): 440-446.

Zhishan, Y., Run E, L., Yanjiang, W. and Xiaoling, S., 2012. The Research on Landslide Disaster Information Publishing System Based on WebGIS. Energy Procedia, 16: 1199-1205.

Zhu, Q., Li, H. and Yang, X., 2009. Hierarchical Semantic Constraint Model for Focused Remote Sensing Information Services. GEOMATICS AND INFORMATION SCIENCE OF WUHAN UNIVERSITY, 34(12): 1454-1457. 